\title{
Performance of the GLAS Space Lidar Receiver through its Seven-Year Space Mission
}

\author{
Xiaoli Sun, Peggy L. Jester, James B. Abshire, Edward S. Chang \\ NASA Goddard Space Flight Center, Code 694/614/690/428, Greenbelt, Maryland 20771, USA \\ xiaoli.sun-1@nasa.gov
}

\begin{abstract}
We report the receiver performance and key components' characteristics of the Geoscience Laser Altimeter System (GLAS) on ICESat after seven years in space as monitored during the mission and tests at the end of mission.
\end{abstract}

\section{Introduction}

NASA's Ice, Cloud, and land Elevation Satellite (ICESat) mission [1,2] carrying the Geoscience Laser Altimeter System (GLAS) Instrument, was launched on January 12, 2003. The three lasers on ICESat have made a total of 1.98 billion laser shot measurements of the Earth's surface and atmosphere during its 17 science data collection campaigns over its seven year operating lifetime. ICESat completed its science mission after the last laser stopped operating in October 2009. The spacecraft was de-orbited on August 30, 2010.

The GLAS instrument carried 3 diode-pumped Q-switched Nd:YAG lasers, which emitted 6-nsec wide pulses at 1064 and $532 \mathrm{~nm}$ at a $40-\mathrm{Hz}$ rate. There are three lidar receiver channels, a $1064 \mathrm{~nm}$ surface altimetry channel, a $1064 \mathrm{~nm}$ cloud backscattering lidar channel, and a $532 \mathrm{~nm}$ cloud and aerosol backscattering lidar channel. The altimetry and cloud backscatter channels used Si avalanche photodiode (APD) operated in analog mode as in the Mars Global Surveyor's Mars Orbital Laser Altimeter [3,4]. GLAS also utilized a number of new technologies and techniques for space lidar, including passively Q-switched diode-pumped Nd:YAG lasers, a 1-m diameter telescope, a temperature tuned etalon optical bandpass filter, Si APD single photon counting detectors, 1 Gsample/sec waveform digitizers, ultra stable clock oscillators, and digital signal processing and detection algorithms [5]. A global position system (GPS) receiver was used to provide the spacecraft position and epoch times.

The ICESat mission provided a unique opportunity to monitor the lidar component performance in the space environment over a multi-year time period. We performed a number of engineering tests periodically to monitor the lidar receiver performance, including receiver sensitivity, timing precision, detector dark noise, etc. A series of engineering tests were also performed after the end of the science mission to evaluate the performance of the spare detector, oscillator, waveform digitizer, and GPS receiver. An experiment was conducted which pointed GLAS to Venus to test the receiver sensitivity to star light and to verify GLAS bore sight with respect to the spacecraft coordinate system. These tests provided unique data to assess the degradation and the rate of change of these key lidar components due to space radiation and aging. They also helped to validate new techniques to operate and calibrate future space lidars.

\section{GLAS Detector Responsivity and Dark Count Change in Space}

We periodically monitored the detector noise level over an orbit period with the laser powered off. For these tests the background detector noise over the night side of the Earth gave a measure of the detector dark noise, and the background detector noise over the sunlit side of the Earth gave a measure of the detector responsivity. The Si APD used in the $1064 \mathrm{~nm}$ channels showed no measurable degradation in dark noise and responsivity. The spare detector which remained off until the end of the science mission showed the same performance. The receiver ranging performance stayed the same, $2-3 \mathrm{~cm}$ standard deviation, through out the mission when tested with the on board laser diode test source. The pulse amplitude fluctuation also remained unchanged, about $5 \%$, for mid-level signal range.

The Si APD single photon counting modules (SPCM) used for the $532 \mathrm{~nm}$ aerosol channel [6] showed no change in responsivity but a steady increase in dark count rates from radiation damage, whether they were powered on or off. Warming up the SPCMs to $23^{\circ} \mathrm{C}$ for more than a month near the end of the mission did not change the rate of degradation. Figures 1 and 2 show the SPCM noise in response to the sunlit Earth and the dark count rate over the seven-year period. 


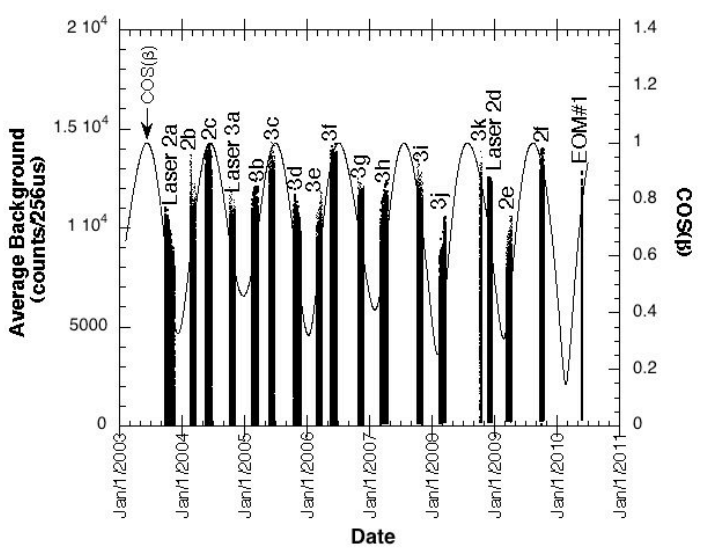

Figure 1. GLAS SPCM output count rates in response to the earth background light since launch. The peak count rate corresponds to the sunlit earth at local noon and gives a measure of the SPCM responsivity after correcting for the sun angle effects.

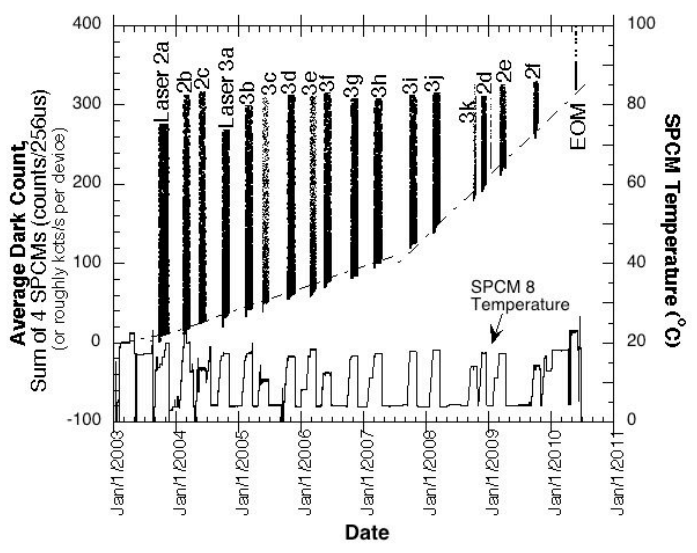

Figure 2. The dark count rate of SPCMs since launch and the trend of the radiation damage. The temperature of one of the SPCM is also plotted. The rate of radiation damage was approximately $60 / \mathrm{s}$ per day per device when the receiver was powered on three times a year and 200/s per day per devices after GLAS was powered on twice a year.

\section{Monitoring Other Lidar Components in GLAS}

There were no significant performance changes in other key lidar receiver components in GLAS, including the prime and spare clock oscillators, analog-to-digital converters, the electronics circuit noise floor, and the prime and spare GPS receivers. The clock oscillator frequency followed a smooth aging trend vs. time. During the end of the mission testing, one of the previously failed lasers, suspected of having a short circuit, was powered up for a final assessment. This action caused an electrical anomaly in the digital to analog converter circuit that the controlled to the signal path switches and set the detector gain became intermittent afterwards.

\section{GLAS Bore Sight Test with Venus}

The GLAS instrument boresight alignment to the spacecraft angular reference was measured by turning the spacecraft away from its normal nadir-pointed view and scanning the GLAS receiver across Venus. This was done twice in a raster pattern on 6/15/2010. Radiometric calculations showed Venus should generate about $0.01 \mathrm{nW}$ and $0.1 \mathrm{nW}$ CW light onto the 532 and $1064 \mathrm{~nm}$ channel detectors, respectively. The $532 \mathrm{~nm}$ SPCM detectors easily detected Venus during both attempts. The $1064 \mathrm{~nm}$ detector also detected Venus, though the response was very weak and could only be discerned when overlaying with the $532 \mathrm{~nm}$ measurements. The test results showed a angular offset, $-0.12^{\circ}$ in X-axis and $-0.044^{\circ}$ in Y-axis, between GLAS receiver field of view and the instrument star tracker, which was consistent with those estimated from the pre-launch tests and in orbit calibration tests.

\section{Acknowledgements}

We thank NASA Headquarters and Earth Science Division for the support of all the end of science mission tests. We thank the GLAS instrument team at NASA Goddard Space Flight Center, and ICESat team at the Ball Aerospace \& Technologies Corporation and University of Colorado Laboratory for Atmospheric and Space Physics for designing and executing special ICESat maneuvers for the end of science mission tests. We also thank the Center for Space Research, University of Texas Austin, for providing the ICESat bore sight alignment analysis for the ICESat to Venus pointing tests.

\section{References}

[1] Zwally, H. J. et al., 'ICESat's laser measurements of polar ice, atmosphere, ocean, and land,' J. Geodyn, Vol. 34, 405-445, 2002.

[2] Schutz, B. E. et al., 'Overview of the ICESat Mission,' Geophys. Res. Lett., Vol. 32, L21S01, 2005.

[3] J. B. Abshire, X. Sun, and R. Afzal, 'Mars orbiter laser altimeter: receiver model and performance analysis,' Applied Optics, Vol. 39, No. 15, pp. 2449-2460, May 2000.

[4] X. Sun, G. A. Neumann, J. B. Abshire, and M. T. Zuber, 'Mars $1064 \mathrm{~nm}$ spectral radiance measurements determined from the receiver noise response of the Mars Orbiter Laser Altimeter,' Applied Optics, Vol. 45, No. 17, June 2006.

[5] J. B. Abshire, X. Sun, H. Riris, M. Sirota, J. McGarry, S. Palm, D. Yi, and P. Liiva, 'Geoscience Laser Altimeter System (GLAS) on the ICESat mission: on-orbit measurement performance,' Geophys. Res. Lett., Vol. 32, L21S02, 2005.

[6] X. Sun X. Sun, M. A. Krainak, W. E. Hasselbrack, Derek F. Sykora, R. La Rue, 'Single photon counting at 950 to 1300 nm using InGaAsP photocathode - GaAs avalanche photodiode hybrid photomultiplier tubes,' Journal of Modern Optics, Vol. 56, pp. 284-295, 2009. 